\title{
Dielectric Slab Mode Antenna for Integrated Millimeter-wave Transceiver Front-ends
}

\author{
Andreas Patrovsky ${ }^{1, *}, \mathrm{Ke} \mathrm{Wu}^{2}$ \\ ${ }^{1}$ was with Ecole Polytechnique de Montréal, now with EADS Deutschland GmbH, Cassidian, 85077 Manching, Germany \\ ${ }^{2}$ Ecole Polytechnique de Montréal, Poly-Grames Research Center, Montreal, Québec, Canada H3T 1J4 \\ *Corresponding Author: AndrPatr@gmail.com
}

Copyright (C) 2013 Horizon Research Publishing All rights reserved.

\begin{abstract}
A novel type of integrated dielectric antenna is presented, which is suitable for low-loss integrated transceiver front-ends in the upper microwave or millimeter wave frequency ranges. The proposed antenna comprises a dielectric high permittivity substrate acting as grounded slab waveguide and a simple planar lens on top for beam focusing. The guided wave is gradually transformed to free space by a curved ground plane for end-fire radiation from the substrate edge. Apart from high radiation efficiency due to very low conductor losses, the use of a standard substrate material also simplifies manufacturing and allows accommodating MMICs or bias circuitry at minimum cost. Simulation and measurement results are presented for a scaled prototype in $\mathrm{X}$-band. Simulation studies were also conducted at millimeter-wave frequencies, where the low-loss advantage is even more evident. Having dimensions of $10 \mathrm{~mm} \times 18 \mathrm{~mm}$, an example design provides a gain of $15 \mathrm{dBi}$ at $60 \mathrm{GHz}$ and a radiation efficiency of more than $80 \%$ if a Duroid ${ }^{\circledR} 6010 \mathrm{LM}$ substrate is used. Good input impedance matching is achieved in a bandwidth of over $20 \%$, covering the entire unlicensed $60-\mathrm{GHz}$ band.
\end{abstract}

Keywords Dielectric Antennas, Hybrid Integration, Lens Antennas, Millimeter Wave Antennas, Slab Waveguides, Transceiver Front-ends, 60-Ghz Band

\section{Introduction}

Among the most important parts of a wireless system is the antenna, since it strongly influences the overall receiver sensitivity and the link budget. In the near future, wireless transmission for consumer products will also happen at much higher frequencies than nowadays, i.e. in the millimeter wave (mm-wave) frequency range. This is required to achieve very fast data exchange and HD video streaming between all kinds of consumer products [1-3]. High radiation efficiency is particularly important due to significant free-space loss, very limited battery capacity in portable devices and consequential low transmit power levels, which is distributed over wide bandwidths. In this context, a multitude of problems arises at higher frequencies, which were not formerly experienced to such an extent in the lower microwave range. Those include high conductor losses and the critical interconnection of a transceiver MMIC to an external antenna. On-chip antennas have other well-known drawbacks. Their radiation efficiency on conductive high permittivity silicon is poor $[4,5]$ and in spite of the short wavelength, they still occupy a non-negligible area on an MMIC chip, which is an important cost factor.

A novel hybrid antenna concept is presented in this paper. (Figure 1) It is suitable to overcome the above-mentioned difficulties. Guidance and beam collimation of a surface wave are achieved by dielectric means to reduce conductor losses to a minimum. Using a planar substrate, this so-called slab mode antenna can be manufactured at low cost by a printed circuit process and is suitable for hybrid integration with active MMICs. Furthermore, the end-fire radiation parallel to the substrate plane complements the usual radiation in perpendicular directions. This may help to increase the overall antenna coverage or increase the throughput in a MIMO multi-antenna system.

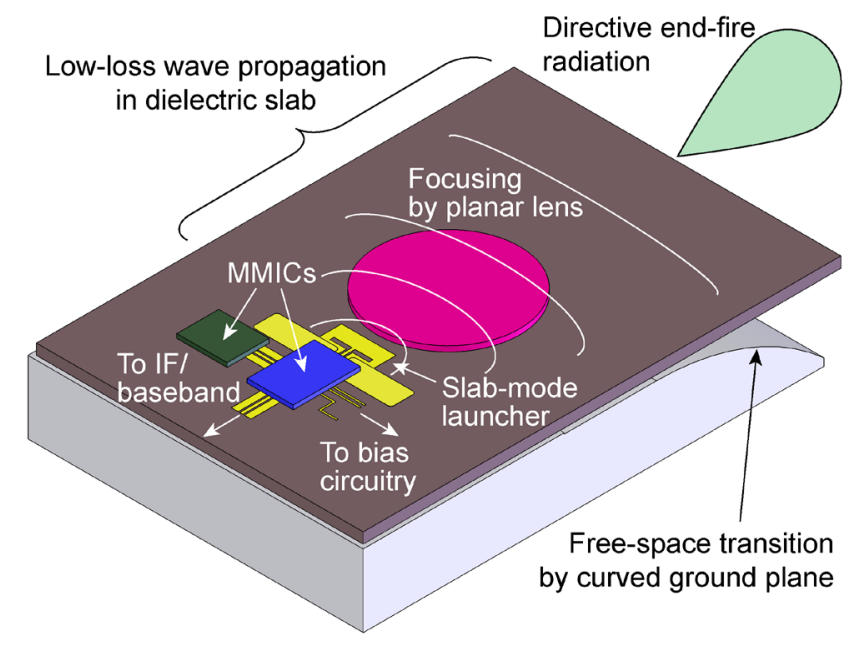

Figure 1. Conceptual assembly of a hybrid integrated slab mode antenna

Section 2 describes the underlying design and operating 
principle. Simulation and measurement results for a fabricated scaled prototype at $12 \mathrm{GHz}$ are shown in section 3 . An additional simulation study of a $60-\mathrm{GHz}$ example antenna is also presented to demonstrate its suitability for mm-wave frequencies.

\section{Method and Concept}

\subsection{Description of the Antenna Parts}

Figure 1 shows a conceptual assembly of the proposed slab mode antenna. The principal part is a planar high permittivity substrate $\left(\varepsilon_{\mathrm{r}} \approx 10\right)$ on a ground plane, which serves as grounded dielectric slab guide for the propagating $T M_{0}$ surface wave. The main electric field component of this mode is normal to the ground plane. Depending on the dielectric material, such a slab guide can have very low transmission loss. Dielectric dissipation is usually the dominant loss mechanism due to the absence of field singularities in the slab guide. An advantageous field distribution leads to low current densities and therefore to low conductor losses in the ground plane. The latter are particularly low if a thin low-permittivity insulation film $\left(\varepsilon_{\mathrm{r}}\right.$ $\approx 2$ ) is introduced between the substrate and ground. Then, the tangential magnetic field intensity right above the ground plane is lowered and current densities are reduced. The $T M_{0}$ slab mode is excited by a coplanar wave launcher. (Figure 2)
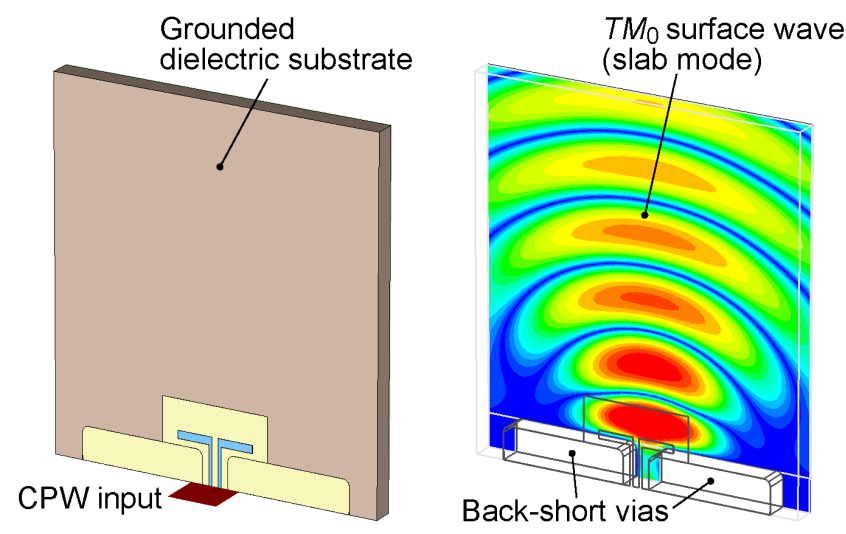

Figure 2. $T M_{0}$ slab mode launcher and electric field visualization

Coplanar waveguide (CPW) was chosen as input line because it is compatible with MMICs and with the relative thick substrate necessary for sufficient field confinement. The substrate thickness $d$ is in the order of one third of a wavelength in the dielectric medium or about $0.5 \mathrm{~mm}$ at 60 $\mathrm{GHz}$ for the exemplary used Duroid ${ }^{\circledR} 6010 \mathrm{LM}$ laminate $\left(\varepsilon_{\mathrm{r}}=\right.$ 10.2) from Rogers ${ }^{\circledR}$ Corp. Such a thickness is still good to handle in a manufacturing process. The launcher's large metallized back-short vias can simultaneously be used as RF or DC ground or for heat dissipation from active MMICs. This kind of wave launcher was first used for a transition from CPW to substrate integrated image guide [6] and is described there in more detail. Comparable $T M_{0}$ slab mode launchers were published by other authors [7,8]. However, the presented design is superior in terms of low parasitic radiation, achievable bandwidth of over $20 \%$, and almost perfect front-to-back ratio due to the reflector-like back-short vias. It was also used in a hybrid integrated mm-wave front-end at $60 \mathrm{GHz}$ [9]. MMIC transceiver front-ends and IF or bias circuits can be mounted on the same substrate that is used for the antenna. (Figure 1) Consequently, the antenna is easy to integrate and cost-efficient. Besides wire-bonding, the flip-chip technique is available to achieve very low interconnect loss to the CPW antenna input. A very short CPW section is feasible and recommended for lowest loss.

The slab mode launcher generates a cylindrical slab wave in the forward half space, which would result in a very wide beam width. A simple planar dielectric lens on top of the substrate is capable of focusing the beam effectively. Field simulations illustrate how the $T M_{0}$ surface wave on the grounded slab is collimated by the planar lens. The guided wave is then gradually transformed into an ungrounded slab mode by the curved ground plane and is finally radiated from the substrate edge in end-fire direction. (Figure 3 )

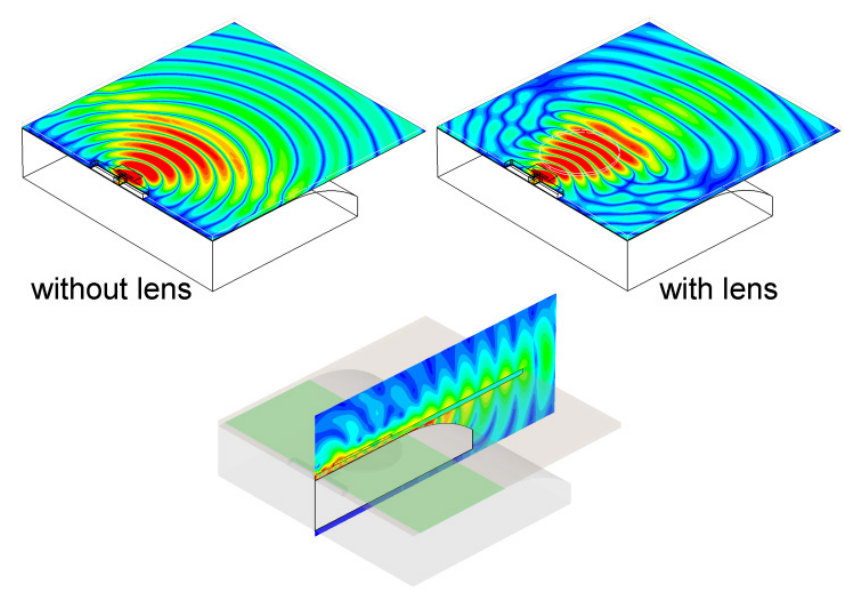

Figure 3. Simulated electric field on horizontal and vertical cut planes

The underlying focusing effect of the planar lens is discussed in the following subsection, along with a quantitative analysis of the propagation constant in the dielectric slab. The necessary curved ground body can be fabricated at low cost by plastic injection molding and subsequent electroplating. It may be part of the housing of a later wireless consumer product. Since the wave is loosely guided in the ungrounded part of the slab and the effective permittivity is low, reflections or scatterings from the substrate edge are negligible. This is in spite of the high refractive index contrast between the substrate material and the surrounding air, which normally causes a strong field trapping effect $[5,10]$. Directive radiation in the vertical plane is achieved as a result of the pronounced vertical field extension that leads to a large effective antenna aperture. The gain can be enhanced further by thinning the substrate towards the end, similar to a dielectric rod or wedge antenna [11]. In the horizontal plane, the effective aperture can be extended by using a larger lens for higher gain and narrower 
beam width.

\subsection{Slab Mode Propagation}

A simple planar dielectric lens on top of the grounded slab guide is capable of collimating the beam in the horizontal plane. This effect can be explained by a local increase in the substrate thickness, which in turn decreases the phase velocity of the guided wave in those zones. The cylindrical phase fronts of the launched surface wave are flattened in this way to augment the antenna gain. A simple but efficient method being available to determine the phase constant in a layered slab guide is the transverse resonance technique $[12,13]$. Its distinct advantage is that boundary conditions at dielectric interfaces can easily be handled as junctions of different transmission lines. The phase constants $\beta$ of the guided modes are obtained by finding the roots of a single characteristic equation. Figure 4 shows the cross-section of the grounded slab guide including a low-permittivity insulation film. Its transverse resonance representation is shown on the right hand side.
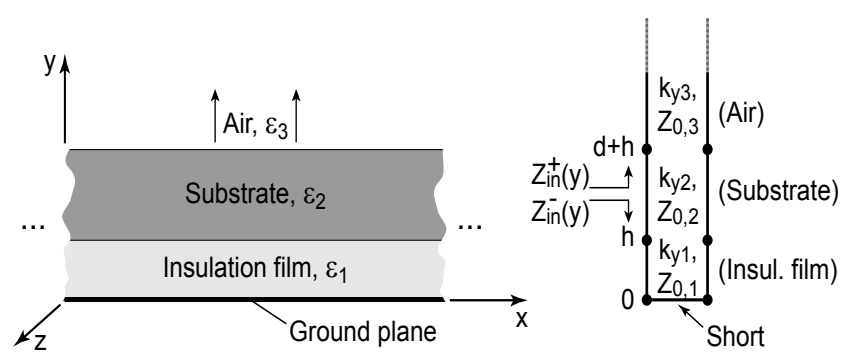

Figure 4. Grounded dielectric slab waveguide (1.) and its transverse resonance representation (r.)

The separability condition

$$
k_{y i}^{2}=k_{i}^{2}-\beta^{2}
$$

holds for the uniform slab waveguide with wave number

$$
k_{i}=\frac{2 \pi f \sqrt{\varepsilon_{r, i}}}{c_{0}}
$$

and transverse wave number $k_{y i}$ in the $i$-th dielectric layer (Cartesian coordinates and uniformity in $x$-direction). Variable $f$ denotes the frequency, $c_{0}$ the speed of light, and $\varepsilon_{r, i}$ is the relative permittivity of the respective layer. The modal fields in a waveguide form standing waves in the transverse plane and therefore the structure can be modeled as a resonant transmission line circuit obeying the following resonance condition

$$
Z_{\text {in }}^{+}(y)+Z_{\text {in }}^{-}(y)=0 \quad \forall y
$$

$Z^{+}{ }_{\text {in }}(y)$ and $Z_{\text {in }}(y)$ are the input impedances seen at a point $\mathrm{y}$ on the resonant transmission line when looking towards the positive or negative y-direction, respectively. (Figure 4) By means of the well-known transmission line impedance equation [13]

$$
Z_{\text {in }}=Z_{0} \frac{Z_{L}+j Z_{0} \tan \left(k_{y} l\right)}{Z_{0}+j Z_{L} \tan \left(k_{y} l\right)}
$$

the impedance transformed by the transmission lines having characteristic impedance $Z_{0}$ and length $l$ can be evaluated at an arbitrary position $y . Z_{L}$ is the load impedance at the end of a transmission line and $Z_{0}$ corresponds to the wave impedance of the $T M$ - or $T E$-modes with transverse propagation constant $k_{y i}$ in the respective dielectric layer [13]:

$$
\begin{gathered}
Z_{0, i}=Z_{T M i}=\sqrt{\frac{\mu_{0}}{\varepsilon_{i}}} \frac{k_{y i}}{k_{i}} \text { for TM-modes } \\
Z_{0, i}=Z_{T E i}=\sqrt{\frac{\mu_{0}}{\varepsilon_{i}}} \frac{k_{i}}{k_{y i}} \text { for TE-modes }
\end{gathered}
$$

Evaluating the resonance condition (3) at $y=h$ with (4) for the interesting fundamental $T M_{0}$ slab mode yields

$$
j Z_{T M 1} \tan \left(k_{y 1} h\right)+Z_{T M 2} \frac{-Z_{T M 3}+j Z_{T M 2} \tan \left(k_{y 2} d\right)}{Z_{T M 2}-j Z_{T M 3} \tan \left(k_{y 2} d\right)}=0
$$

if layer " 3 ", the air layer, is approximated to be infinite. Applying the substitutions (1), (2), and (5) to equation (7) results in the final characteristic equation, which allows the calculation of $\beta$ by a root-finding algorithm for a given frequency. Several roots may exist, corresponding to the fundamental and higher order TM modes. Equation (7) can easily be extended to more than three dielectric layers, e.g. if the collimating lens and the substrate consist of different materials or if the effects of a potential air gap should be analyzed. The transverse resonance technique described above was used to compute the normalized phase constant of the fundamental $T M_{0}$ mode for the case that substrate and lens are made from Duroid ${ }^{\circledR} 6010$ LM high frequency laminate. (Figure 5)

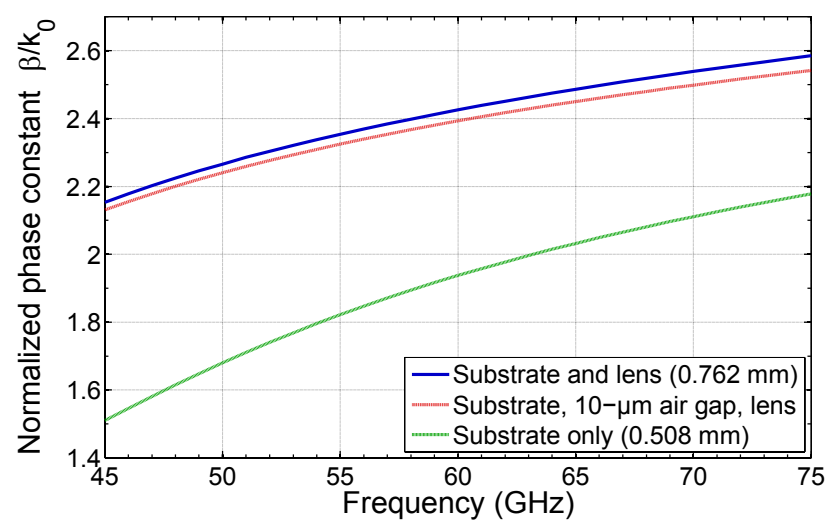

Figure 5. Normalized phase constants of the fundamental $T M_{0}$ slab mode

In the region with superimposed lens, corresponding to an increased substrate thickness if the lens is of the same material, the guided wavelength $\lambda_{g}=2 \pi / \beta$ is shorter than on 
the substrate alone. The resulting phase equalization is responsible for the collimating effect. It is observed that the sensitivity to a small air gap between the lens and the substrate is low, so that certain surface irregularities can be tolerated. Once those parameters are known, the shape and size of a suitable lens can be determined. Planar lens design is covered elsewhere, e.g. [14,15]. Well-known numerical optimization techniques can be applied to the lens design to obtain the desired antenna characteristics (e.g. maximum gain or minimum side lobes). Simple circular lenses are used in the following examples of medium gain slab mode antennas.

\section{Results}

\subsection{Prototype at $12 \mathrm{GHz}$}

A prototype of the antenna described in the previous section was designed and realized in the X-band at around 12 GHz. (Figure 6) Its dimensions are listed in Table 1.

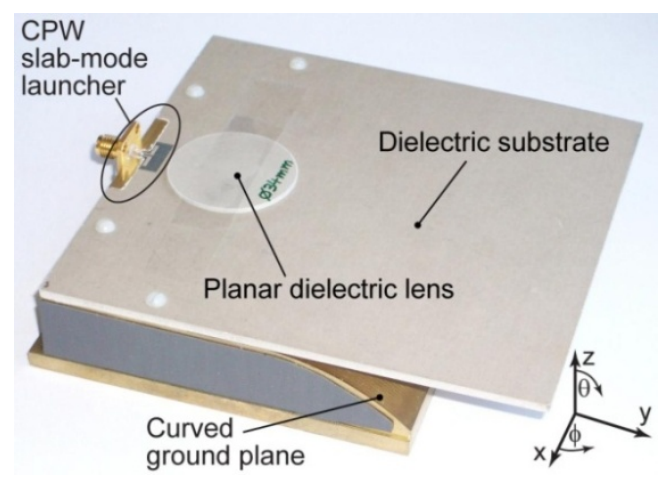

Figure 6. Slab mode antenna prototype

Table 1. Dimensions of the slab-mode antenna prototype at $12 \mathrm{GHz}$.

\begin{tabular}{ccc}
\hline Variable & Dimension & Value $(\mathrm{mm})$ \\
$\mathrm{a}$ & Substrate length & 120 \\
$\mathrm{~b}$ & Substrate width & 120 \\
$\mathrm{~d}$ & Substrate thickness & 2.54 \\
$\mathrm{~h}$ & Air gap height (below substrate) & 0.254 \\
$\mathrm{t}$ & Lens thickness & 1.27 \\
$\mathrm{D}$ & Lens diameter & 34 \\
$\mathrm{r}$ & Curved ground plane radius & 60 \\
l & Curved ground plane horiz. length & 40 \\
\hline
\end{tabular}

The curved ground body of the antenna was milled out of a brass block. It also includes the back-short vias of the slab mode launcher and spacers, which form a thin air gap instead of the previously mentioned insulation film. A Duroid ${ }^{\mathbb{B}}$ 6010LM substrate with notches for the vias was fixed thereon. The CPW line and the patch resonator of the launcher were fabricated in printed circuit manner. The patch and the vias were conductively connected with silver epoxy and a standard SMA connector was mounted as an interface to the measurement equipment. The circular lens was cut out of another Duroid substrate and was fixed with adhesive tape on the grounded slab section of the antenna.

The input reflection coefficient was measured by means of a network analyzer at the SMA connector. It is compared to simulation results obtained with the 3D time-domain solver of CST Microwave Studio ${ }^{\circledR}$. (Figure 7) Some degradation is caused by the SMA connector, which was not taken into account in the simulation. In addition, a degradation of the input matching and shift to lower frequencies may be caused by fabrication tolerances, because the alignment of the milled metal body with the substrate was not absolutely precise. Nevertheless, the obtained bandwidths for $>10 \mathrm{~dB}$ return loss are about the same.

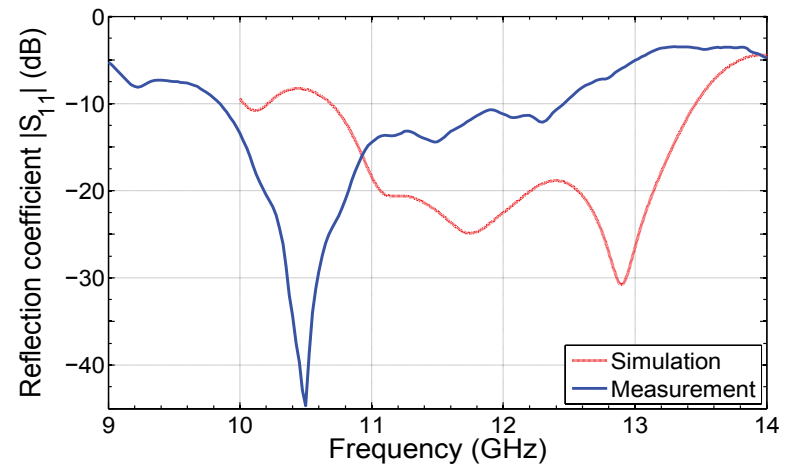

Figure 7. Measured and simulated reflection coefficient of the prototype

Far field radiation patterns were measured at $12 \mathrm{GHz}$ in an anechoic chamber. (Figure 8)
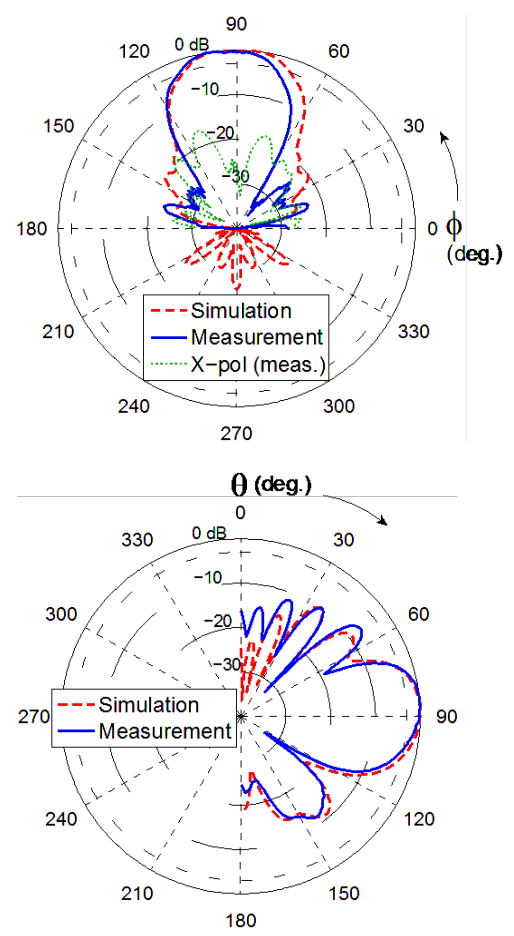

Figure 8. Normalized far field radiation patterns of the prototype antenna in the horizontal H-plane (top) and in the vertical E-plane at $12 \mathrm{GHz}$.

Simulated and measured patterns agree well. The half-power beam width is $33^{\circ}$ in the H-plane and $36^{\circ}$ in the 
E-plane. The measured gain of $12.0 \mathrm{dBi}$ is slightly lower than the simulated value of $12.6 \mathrm{dBi}$ at an overall measurement uncertainty of $\pm 0.6 \mathrm{~dB}$. Increased side lobes are observed in the E-plane as a consequence of the superposition of parasitic radiation by the slab mode launcher and the intended beam. If desired, this side effect can be reduced significantly by use of absorber paint or resistive shielding in a later housing. The fraction of power which is radiated by the slot dipole to free space instead of being coupled to the surface wave can be approximated by $\varepsilon_{r, S}{ }^{-3 / 2}[6,16]$, where $\varepsilon_{r, S}$ is the relative permittivity of the substrate. As a consequence, only high permittivity substrates should be used together with this kind of slab mode launcher, since otherwise too much power is radiated uncontrolled.

The slab mode antenna was built for operation at $12 \mathrm{GHz}$ to prove the operating principle and to confirm the simulated antenna characteristics. It was not optimized for compactness. The advantages of this type of antenna, in particular the high radiation efficiency, become apparent at higher frequencies only, where conductor losses are generally more dominant. For this reason, an example antenna design was studied theoretically at $60 \mathrm{GHz}$. Results are presented in the following section.

\subsection{0-GHz Example Antenna}

Another slab mode antenna was designed for operation in the unlicensed $60-\mathrm{GHz}$ band. (Figure 9)

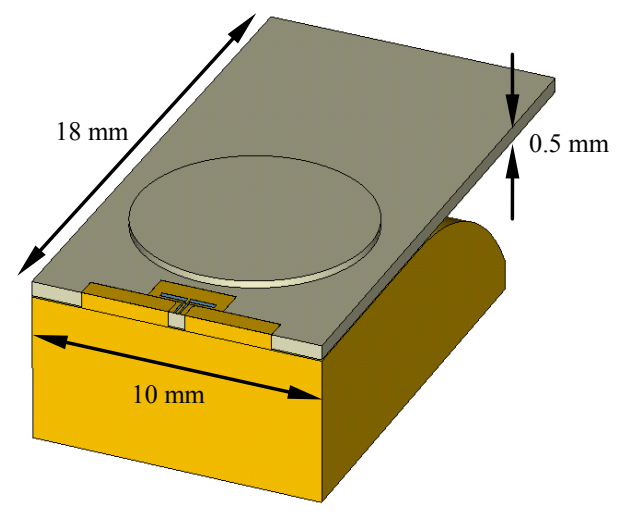

Figure 9. Slab mode antenna design for the $60-\mathrm{GHz}$ frequency range
In this case, the design parameters were (description see Table 1): $a=18 \mathrm{~mm} ; b=10 \mathrm{~mm} ; d=0.508 \mathrm{~mm} ; h=0.05 \mathrm{~mm}$; $t=0.254 \mathrm{~mm} ; D=8 \mathrm{~mm} ; r=8 \mathrm{~mm} ; l=7 \mathrm{~mm}$. The air gap of the previous prototype antenna was replaced by a PTFE insulation film $\left(\varepsilon_{\mathrm{r}}=2.08 ; \tan \delta=0.001\right)$. Again, Duroid $^{\circledR} 6010 \mathrm{LM}$ laminate was used for the slab and the lens, having a loss tangent $\tan \delta=0.003$. All conductors were modeled with specific conductivity $\sigma=2.0 \cdot 10^{7} \mathrm{~S} / \mathrm{m}$. Good input return loss $>15 \mathrm{~dB}$ was achieved for an operating bandwidth of more than $20 \%$. (Figure 10) This large bandwidth easily covers the entire unlicensed $60-\mathrm{GHz}$ band.

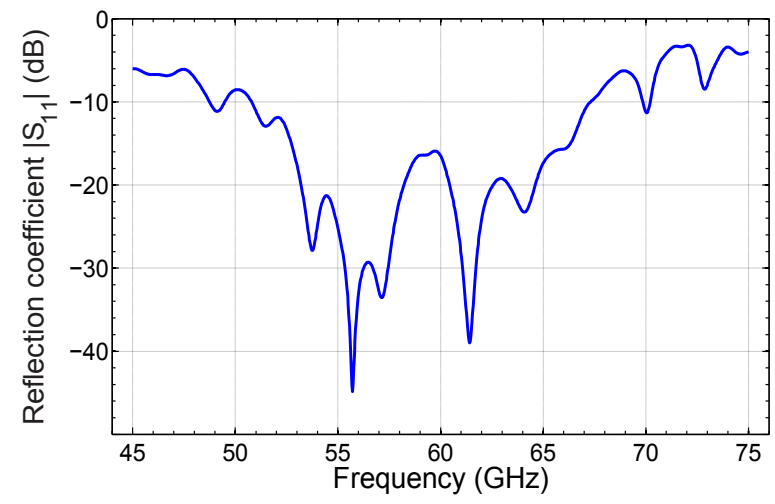

Figure 10. Simulated reflection coefficient of the $60-\mathrm{GHz}$ slab mode antenna

Radiation pattern cuts in the $\mathrm{H}$ - and E-plane at $57 \mathrm{GHz}, 60$ $\mathrm{GHz}$, and $63 \mathrm{GHz}$ show excellent frequency stability. (Figure 11) In this frequency range, the simulated gain varies between $14.0 \mathrm{dBi}$ and $15.3 \mathrm{dBi}$ in main direction. The H-plane beam width is somewhat narrower at higher frequencies. At $60 \mathrm{GHz}$, the H-plane half-power beam width is $22^{\circ}$, but it can be customized by the size of the lens. A very wide beam width is obtained if no collimating lens is used at all, which results in lower gain but wider coverage, however. For this case, the antenna size can be made very compact. The E-plane half-power beam width is $37^{\circ}$. It can be altered by changing the length and the taper of the substrate cantilever overlapping the ground body, similar to a dielectric rod or wedge antenna [11]. 

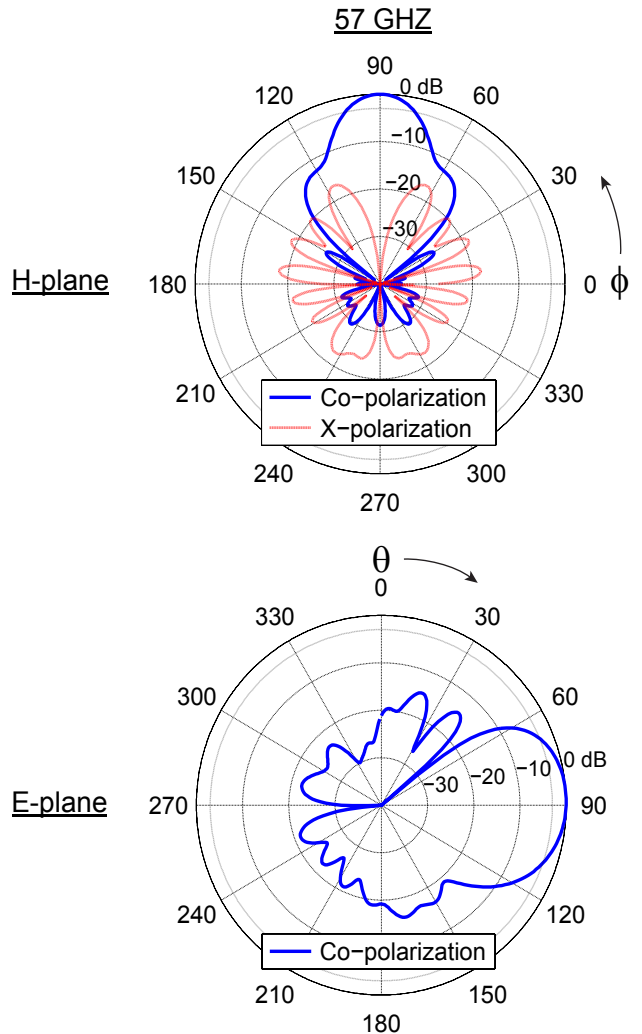

Directivity: $14.7 \mathrm{~dB}$

Gain: $14.0 \mathrm{dBi}$

Radiation efficiency: $85.4 \%$
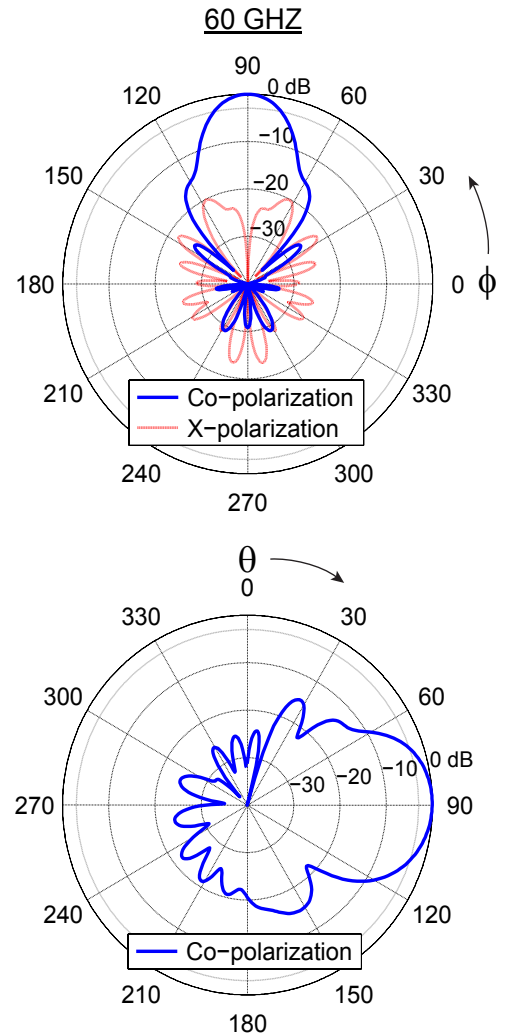

Directivity: $15.8 \mathrm{dBi}$

Gain: $15.1 \mathrm{dBi}$

Radiation efficiency: $84.3 \%$
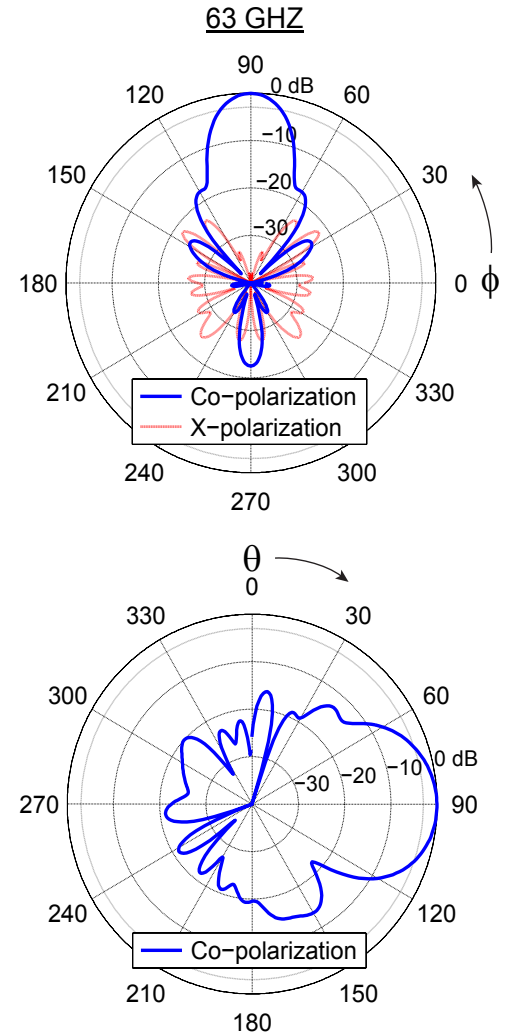

Directivity: $16.1 \mathrm{dBi}$

Gain: $15.3 \mathrm{dBi}$

Radiation efficiency: $82.4 \%$

Figure 11. Simulated far field radiation patterns (normalized) of the 60-GHz slab-mode antenna in the H- and E-planes

Based on the given material specifications, the radiation efficiency of the $60-\mathrm{GHz}$ antenna design is better than $80 \%$. In a second trial simulation, all metallic parts were modeled as perfect electric conductor, i.e. only dielectric losses were taken into account. In this test, the radiation efficiency rises by only $3 \%$ compared to the lossy conductor. This confirms the very low conductor loss of the slab mode antenna and suggests its use for even higher frequencies. Measurement results are not available due to the limited micro-fabrication capabilities in the university laboratory and because of the great difficulties with connecting the mm-wave measurement equipment for radiation pattern measurements in an anechoic chamber. Nevertheless, the presented simulation results are deemed accurate because simulation and measurement at $12 \mathrm{GHz}$ also agreed well.

Similar to [15], the feature of beam scanning is feasible in the horizontal plane by arranging several switched slab mode launchers around the collimating lens.

\section{Conclusion}

The developed dielectric slab mode antenna is most suitable for applications at the upper end of the microwave range or in the mm-wave range. This is because of its low-loss dielectric construction, its wide operating bandwidth, and its customizable gain in a range of about 10
$\mathrm{dBi}$ to $20 \mathrm{dBi}$. Other beneficial features include the use of a planar substrate to promote low-cost manufacturing and the compatibility to MMICs which can be mounted in flip-chip manner for lowest interconnection loss at very high frequencies. The design relies on high permittivity substrates with $\varepsilon_{\mathrm{r}}>8$, unless a wave launcher is developed which can deal with lower permittivity. Most other integrated antennas perform poor on high permittivity substrates due to field trapping effects, so that the slab mode antenna provides an alternative. It is furthermore compatible to relative thick substrates, which are easier and therefore cheaper to handle during production. Radiation occurs in end-fire direction, i.e. parallel to the substrate plane, which may be desired in many cases of product integration. There are very few antenna designs having the same characteristic. Due to its simple construction and very low conductor loss, the dielectric slab mode antenna can be a practicable option up to terahertz frequencies.

\section{Acknowledgements}

The authors like to thank Steve Dubé for fabricating the prototype. A special thanks is extended to Rogers Corp. for providing the Duroid ${ }^{\mathbb{B}} 6010 \mathrm{LM}$ substrates at no charge. 


\section{REFERENCES}

[1] D. R. Vizard. Millimeter-wave applications: from satellite communications to security systems, Microwave Journal, Vol.49, No.7, 22-36.

[2] P. Smulders. Exploiting the $60 \mathrm{GHz}$ band for local wireless multimedia access: prospects and future directions, IEEE Communications Magazine, Vol.40, No.1, 140-147.

[3] J. Laskar, S. Pinel, D. Dawn, S. Sarkar, B. Perumana, P. Sen, The next wireless wave is a millimeter wave, Microwave Journal, Vol.50, No.8, 22-32.

[4] Y. P. Zhang, M. Sun, L. H. Guo, On-chip antennas for $60-\mathrm{GHz}$ radios in silicon technology, IEEE Transactions on Electron Devices, Vol.52, No.7, 1664-1668.

[5] A. Babakhani, G. Xiang, A. Komijani, A. Natarajan, A. Hajimiri, A 77-GHz phased-array transceiver with on-chip antennas in silicon: receiver and antennas, IEEE Journal of Solid-State Circuits, Vol.41, No.12, 2795-2806.

[6] A. Patrovsky, K. Wu, 94-GHz broadband transition from coplanar waveguide to substrate integrated image guide (SIIG), IEEE MTT-S International Microwave Symposium, Honolulu, HI, USA, 1551-1554, 2007.

[7] A. R. Perkons, Y. Qian, T. Itoh, TM surface-wave power combining by a planar active-lens amplifier, IEEE Transactions on Microwave Theory and Techniques, Vol.46, No.6, 775-783.

[8] H. F. Hammad, Y. M. M. Antar, A. P. Freundorfer, S. F. Mahmoud, Uni-planar CPW-fed slot launchers for efficient TM0 surface-wave excitation, IEEE Transactions on Microwave Theory and Techniques, Vol.51, No.4,
1234-1240.

[9] A. Patrovsky, K. Wu, Active $60 \mathrm{GHz}$ front-end with integrated dielectric antenna, Electronics Letters, Vol.45, No.15, 765-766.

[10] D. H. Evans, P. J. Gibson, A coplanar waveguide antenna for MMICs, 18th European Microwave Conference, Stockholm, Sweden, 312-317, 1988.

[11] G. M. Whitman, C. Pinthong, C. Wan-Yu, F. K. Schwering, Rigorous TE solution to the dielectric wedge antenna fed by a slab waveguide, IEEE Transactions on Antennas and Propagation, Vol. 54, No.1, 101-114.

[12] R. Sorrentino, Transverse resonance technique, in Numerical Techniques for Microwave and Millimeter-Wave Passive Structures, chapter 11, John Wiley \& Sons, New York, 1989.

[13] D. M. Pozar, Microwave Engineering, 3rd edition, John Wiley \& Sons, New York, 2005.

[14] L. C. Gunderson, An electromagnetic analysis of a cylindrical homogeneous lens, IEEE Transactions on Antennas and Propagation, Vol.20, No.4, 476-479.

[15] B. Schoenlinner, W. Xidong, J. P. Ebling, G. V. Eleftheriades, G. M. Rebeiz, Wide-scan spherical-lens antennas for automotive radars, IEEE Transactions on Microwave Theory and Techniques, Vol.50, No.9, 2166-2175.

[16] N. G. Alexopoulos, P. B. Katehi, D. B. Rutledge, Substrate optimization for integrated circuit antennas, IEEE Transactions on Microwave Theory and Techniques, Vol.31, No.7, 550-557. 